medicine safety of parenteral ready-to-use all-in-one mixtures, e.g. TPN bags in neonatology.

No conflict of interest.

\section{TCH-046 THE ADVANTAGES OF UV-RAMAN SPECTROSCOPY FOR CHECKING THE STRENGTH OF NALBUPHINE PREPARATIONS}

doi:10.1136/ejhpharm-2013-000276.237

'F Nardella, 'P Collart-Dutilleul, ' $\mathrm{C}$ Weyant, 'L Perello, ${ }^{2} \mathrm{AC}$ Gairard-Dory, ${ }^{3} \mathrm{~B}$ Gourieux, 'G Ubeaud-Sequier. 'Hôpitaux Universitaires de Strasbourg, Laboratoire de Contrôle Service Pharmacie - Pôle Pharmacie Pharmacologie, Strasbourg, France; ${ }^{2}$ Hôpitaux Universitaires de Strasbourg, UF Pharmacotechnie - Service Pharmacie - Pôle Pharmacie Pharmacologie, Strasbourg, France; ${ }^{3 H o ̂ p}$ itaux Universitaires de Strasbourg, Service Pharmacie - Pôle Pharmacie Pharmacologie, Strasbourg, France

Background A paediatric nalbuphine formulation is prepared in the hospital pharmacy of the Nouvel Hôpital Civil of Strasbourg. It was previously checked by HPLC. Following the acquisition of an UV-Raman spectrometer, a method was developed in order to improve the monitoring of nalbuphine preparation.

Purpose To cheque paediatric nalbuphine formulations with a simple, fast and reliable method by using UV-Raman spectroscopy. Materials and Methods In order to validate a method using the QC-prep (a UV-Raman spectrometer), we prepared three concentration ranges, prepared by diluting three different samples of nalbuphine reconstituted in $0.9 \% \mathrm{NaCl}$. Each range was composed of 5 points of calibration. The linearity was validated from the average of the three ranges. The fidelity of the method is tested by repeatability (one solution was sampled five times by the QC-prep) and reproducibility (five different solutions were sampled at one time). The method is considered as valid if the linearity is good enough $\left(\mathrm{r}^{2}>0.999\right)$ and the coefficient of variation $(\mathrm{CV})$ and relative error of repeatability and reproducibility are below $5 \%$.

Results The QC-prep method for nalbuphine $1 \mathrm{mg} / \mathrm{ml}$ in $0.9 \%$ $\mathrm{NaCl}$ is valid in terms of:

- Linearity: the calibration is linear from 0.2 to $2.0 \mathrm{mg} / \mathrm{mL}$ $\left(r^{2}=0.9997\right)$

- Repeatability: the CV is less than $0.25 \%$

- Reproducibility: the CV is less than $2.5 \%$

- Accuracy: the relative error is less than $5 \%$

Five different batches have been checked in routine work. No mistakes have been identified, either in the concentration of the drug (quality control and sample), or in identification of the solvent.

Conclusions Calibration of the QC-prep is simple thanks to easyto-use software. This is a powerful tool that enables us to determine the concentration of nalbuphine more quickly, easily and safely than the HPLC method previously used. The UV-Raman spectroscopy method could be extended to the analysis of other formulations such as paediatric antibiotics preparations.

No conflict of interest.

\section{TCH-047 THE EFFECT OF A ROBOTIC UNIT DOSE DRUG DISPENSING SYSTEM ON MEDICINES ADMINISTRATION ERRORS AND THE COST OF DRUG DISPENSING}

doi:10.1136/ejhpharm-2013-000276.238

M Viprey, C Burgos Leon-Djian, X Dode, G Aulagner. Groupement Hospitalier Est, Pharmacy, Bron Cedex, France

Background A Unit Dose Drug Dispensing System (UDDDS) by a robot (PillPick system, Swisslog) with daily pharmaceutical monitoring of medical prescriptions is being implemented in our hospital, to gradually replace the ward stock distribution system (WSDS), which allowed a low level of pharmaceutical monitoring In 2011, UDDDS was used for 374 beds. UDDDS allows named "ready-to-use" treatments to be dispensed daily, avoiding nurse preparation of pillboxes, necessary with WSDS.

Purpose To assess the impact of a robotic UDDDS on the incidence of medicines administration errors and to assess the cost of this system.

Materials and Methods Medication errors were measured using a direct observation process in two phases, before and after implementation of the UDDDS, in a 23-bed adult cardiology unit with WSDS, computerised prescription order entry and computerised medicines administration record (CristalNet). The cost study took into account both the payroll cost (pharmaceutical staff, nurses) and the cost of the robot. A monthly cost per hospital bed supported was calculated for each system.

Results A total of 3233 medicines administrations were observed (1471 pre-implementation and 1762 post-implementation) for 185 patients (91 pre-implementation and 94 post-implementation). After the introduction of UDDDS the percentage of medicines administration discordances with the medical prescription fell $(46 \%$ to $18 \%$ ). The identification of drugs by nurses improved (18\% to $1 \%)$. The monthly cost was estimated at $€ 142$ per bed with WSDS and at $€ 161$ per bed with UDDDS. Considering the distribution of depreciation and maintenance costs over 950 beds, we assume that the systems costs will become comparable.

Conclusions Unit Dose Drug Dispensing by a robot is comparable to WSDS in terms of cost, while being safer, thanks to automated drug picking and pharmaceutical monitoring of medical prescriptions. Barcode verification technology is advancing.

No conflict of interest.

\section{TCH-048 THE SECURITY OF PHARMACOKINETIC INFORMATION IN ELECTRONIC HEALTH RECORDS}

doi:10.1136/ejhpharm-2013-000276.239

A Sendra García, 0 Ruiz Millo, M Fernández Álvarez, A Moratalla, NV Jimenez Torres, M Climente Martí. Hospital Universitario Dr. Peset, Pharmacy, Valencia, Spain

Background Accurate and complete electronic health record (EHR) information is essential for patient safety, especially when drugs with a narrow therapeutic range are involved.

Purpose To evaluate the quality and quantity of information recorded in EHRs concerning pharmaceutical interventions (PIs) generated by therapeutic drug monitoring (TDM).

Materials and Methods For 6 months, all onco-haematology inpatients were evaluated who were receiving vancomycin ( $\geq 3$ doses). Renal function (RF) was classified into four categories: severe, moderate and mild renal impairment (RI) and normal RF for creatinine clearance (by Cockcroft-Gault equation) $<10$, 10-50, 50-90, $>90 \mathrm{ml} / \mathrm{min}$, respectively. PIs were classified into three categories of importance (high, moderate and low) according to the pharmacotherapy follow-up and the relation between plasma concentration and optimal therapeutic range.

The completeness of EHRs regarding the RF and TDM process (ordering, result and PI-related parameters) was assessed.

A binary logistic regression with odds ratio $(\mathrm{OR})$ was performed using SPSS v.15.0.

Results TDM was performed for 39 (81\%) of 48 patients receiving vancomycin. The median age was 57 years (95\%CI: $52-62) ; 26$ were male $(68 \%)$; 21(54\%) had mild to moderate RI.

There were 76 PIs [median 2/patient (IOR: 2)], 51(67\%), 4 (5\%) and $21(28 \%)$ of high, medium and low importance, respectively; $67(88 \%)$ were accepted. 